\title{
Capítulo I \\ El terrorismo no es un fenómeno nuevo, el concepto sí lo es
}

El terrorismo como práctica es antiguo. Lo nuevo son sus capacidades de destrucción, organización y de sumar adeptos por todo el globo a costos muy bajos.

MerKe (2005).

$\mathrm{U}$

n fenómeno, según la Real Academia de la Lengua Española, “es toda manifestación que se hace presente a la consciencia de un sujeto y aparece como objeto de su percepción" (Real Academia de la Lengua Española, 2014). Los regímenes internacionales responden a la convergencia de fenómenos que codifican el comportamiento y actividad de los distintos actores en el sistema internacional; son ellos quienes, de manera categórica y al estilo del liberalismo clásico, determinan las causas plausibles y conductas aceptadas sobre las buenas prácticas de coexistencia.

Un concepto, bajo la definición de la Real Academia, es la idea que concibe o forma el entendimiento. $Y$ en esta medida, el concepto de terrorismo es la categoría que intenta idear una forma amorfa. De ahí se desprende todo un cúmulo de apreciaciones y reflexiones cuestionables, controversiales y debatibles que repercutirán en materia fenomenológica y conceptual frente al gran desafío del derecho internacional. 
El análisis académico contemporáneo — desarrollado por disciplinas como el derecho internacional, las relaciones internacionales, la seguridad y la defensa- coincide al señalar que el fenómeno del terrorismo es una nueva amenaza. Esto debido a que en la agenda de seguridad de los Estados y en la arquitectura internacional, el terrorismo ha copado un lugar especial en materias donde antes no tenía tanto protagonismo. Así las cosas, los diferentes actores del sistema internacional han hecho del terrorismo lo que entienden por el mismo, y sobre todo lo que en muchas ocasiones se acomoda a su dimensión estructural y coyuntural de interés.

Todo indica que el terrorismo es la amenaza "de moda", pero lo que no se ha escrito hasta el momento, es que siempre ha sido la amenaza "de moda". Dicho esto, es importante decantar los elementos propios del terrorismo como un factor fundamental en el desenvolvimiento y desarrollo de algunas cuestiones del derecho internacional. El terrorismo es uno de los fenómenos que ha logrado, a pesar de su capacidad destructora, poner al máximo tope a los operadores, regímenes y tomadores de decisiones en materia jurídica, política y de seguridad. Sin embargo, los intentos por precisar su génesis y explicar su evolución teórica y en ocasiones sistémica — desde la mitología griega pasando por la noción de terror en la Revolución Francesa, la muerte del archiduque Francisco Fernando hasta la nueva dimensión con el 11 de septiembre de 2001 y el surgimiento del grupo del Estado Islámico en 2014, entre otros-, han logrado cegar la lógica de su funcionamiento, naturaleza y comportamiento como régimen en la arena internacional.

La palabra terrorismo tiene una dimensión etimológica bastante simple. "Terrorismo" deriva de los vocablos latinos terrere que alude a estremecerse y deterrereo "asustarse de" (Torres, 2012). Desde esa dimensión, a simple vista y para cualquier desprevenido analista, sería bastante fácil determinar lo que es el concepto. No obstante, las dificultades no solo son semánticas y etimológicas sino estructurales, jurídicas, disciplinarias y hasta de seguridad.

En virtud de lo anterior, es complejo identificar un periodo gestacional y embrionario del fenómeno. No obstante, uno de los primeros intentos de registro son los pasajes míticos de la Grecia Antigua. 
En el antiguo mito del Minotauro se detecta una analogía al terrorismo. En la historia clásica, el hombre con cabeza de toro es considerado un ser malévolo que acosaba al pueblo griego y en especial a la isla de Creta y a los atenienses. Según Ovidio, sembraba terror y certidumbre que cualquiera podía ser su próxima víctima. Aunque era producto de una unión material entre los hombres y los dioses, los humanos lo desdeñaban porque atentaba contra su seguridad y la de lo que se conoce ahora como Estado. Teseo se volvió un héroe en ese contexto, al neutralizar al tipo de terrorista que representaba el Minotauro (Aguilar, 2001, p. 15).

El anterior pasaje logra identificar un cierto momento de referencia frente al fenómeno. En ese orden de ideas, todo indica que la amenaza del terrorismo fue primero que la misma noción jurídica y de constructo social de Estado. Las amenazas son las causas estructurales de los sistemas y regímenes para prevenirla. En otras palabras, es gracias a amenazas como el terrorismo, que se ha construido un complejo andamiaje que incluye la costumbre y el tejido jurídico internacional para hacerles frente.

El fenómeno del terrorismo es tan antiguo como la historia de la sociedad humana, y se ha manifestado bajo formas tan diversas como el asesinato político o la toma de rehenes (Owada, 2009), entre otras. Por cuestiones de devenir histórico e interacción de actores no es una nueva amenaza; simplemente ha sobrevivido a la historia bajo una naturaleza mutante y adaptable. Y quizá por eso es que en sus formas más primitivas no logró llamarse “terrorismo" pues, en últimas, ese concepto es nuevo y proviene de una invención moderna occidental (Molano, 2012), aunque esto no significa que la amenaza sea nueva. En consecuencia, desde el derecho y las ciencias sociales contemporáneas ha existido un gran interés por definir y conceptuar su entorno; y es así que la palabra terrorismo surge asociada al Estado y como nombre de un periodo revolucionario bajo la hegemonía de Robespierre (Aznar, 2015).

El término "terror" apareció por primera vez en el léxico político práctico para definir, y en principio no de forma negativa, el régimen excepcional mantenido por el Comité de Salud Pública de abril 
de 1793 a julio de 1794. En contraste, el concepto de "terrorismo" surgió en la etapa thermidoriana de la Revolución Francesa como un término despectivo referido al sistema de gobierno desplegado por la Convención. La palabra "terrorismo" figuró desde 1798 en el Dictionnaire de l'Académie Française, donde quedó fijado como “système, régime de terreur”, en un sentido peyorativo del que carecía antes de Thermidor. El concepto ingresó en el lenguaje político inglés en 1795 como “Government by intimidation” o "A policy intended to strike with terror those against whom it is adopted" (Oxford English Dictionary). Por aquel entonces, el terror era entendido en exclusiva como un régimen, o como una práctica característica del poder estatal, cuya virtualidad era recurrir de forma sistemática a la violencia contra personas y cosas, provocando de ese modo un ambiente de temor generalizado (González, 2014, p. 124).

La constante antigua y moderna de la fenomenología de la amenaza es la que la ha llevado a perdurar en el tiempo. El terrorismo es el uso sistemático de la intimidación coercitiva, por lo general al servicio de intereses políticos. Pero no es una filosofía ni un movimiento: es un método (Wilkinson \& Mari, 2008). Para otros autores, el concepto compromete una complejidad mucho mayor, por ejemplo, que el terrorismo es un instrumento privilegiado de la revolución (refiriéndose a la Revolución Francesa) y que forma parte de la desobediencia civil, de la crítica, del instrumental político de la ideología de la revolución (Martínez, 2014). No obstante, también hay otro tipo de aproximación etimológica con la que el terrorismo se configura como un tipo determinado de violencia que genera efectos psíquicos desproporcionados respecto a sus consecuencias materiales (Aparicio, 2009).

En ese sentido, más allá del panorama académico, siguiendo el patrón definicional el Senado francés también se ha valido de una concepción terminológica para saber bajo qué condiciones determina lo que el país entiende por terrorismo; así las cosas, en Francia el Código Penal define el acto terrorista como un acto relacionado con "una empresa individual o colectiva para el orden público que perturben gravemente por la intimidación o el terror" (Sénat, 2014). 
De esa manera, la transversalidad de la amenaza ha tenido un flujo constante de niveles de intensidad en diferentes momentos de la historia. Uno de los trabajos pioneros que ha intentado explicar de forma sistémica la lógica de la existencia del terrorismo global es el de David Rapoport. Según él, el terrorismo puede distribuirse en cuatro oleadas que se caracterizan por tener elementos comunes y compartidos en la detentación de las actividades de grupos u organizaciones (Molano, 2012).

La primera de ellas es la "creación de la doctrina" o la "revolucionaria anarquista". Esta primera etapa, enmarcada como enfoque epistemológico, logra determinar que a partir de 1870 hasta aproximadamente el inicio de la Primera Guerra Mundial sucede el fenómeno de la "nueva comunicación" (Rapoport, 2004). Esta ola identifica la táctica del magnicidio como instrumento en la generación de terror. La organización anarquista rusa Narodnaya Volya (Voluntad del Pueblo) imparte, en 1879 , las primeras acciones con el objetivo de "despertar la conciencia de las masas" hacia cambios radicales (DerGhounkassian, 2013).

Esta ola es el punto de partida de la modernidad del concepto. Articula la noción del terror en contra de las instituciones con el propósito de atacarlas y destruir la convencionalidad de las mismas. Generó una condición de doctrina que, según Stepniak ${ }^{2}$, al describir el terrorismo ruso sentenciaba que el terrorista era "noble, terrible, fascinantemente irresistible y podía unir las dos cosas sublimes de la grandeza humana, el ser mártir y héroe al mismo tiempo" (Rapoport, 2004, p. 46).

El segundo periodo u ola data de los años veinte a los sesenta del siglo XX. Se denomina "la anti-colonial". En esta etapa se resalta la expresión de la lucha por la autodeterminación de los pueblos colonizados por las potencias occidentales (DerGhounkassian, 2013) y se exacerban los sentimientos separatistas, antiimperialistas y nacionalistas llevándolos a su máxima expresión mediante el uso de explosivos. En esta ola es relevante mencionar que luego de culminada la Segunda Guerra Mundial, la reconfiguración del sistema internacional estuvo influida por el carácter de las potencias vencedoras. Fenómeno

2 Fue uno de los principales protagonistas en la historia del terrorismo ruso, quien adquirió fama al asesinar al director de la policía secreta de Rusia como represalia por la ejecución de Ivan Kovalsky, en el año 1878. 
que trajo consigo una serie de manifestaciones en la periferia que, a su vez, generaron un proceso de emancipación concreta. A pesar de esto, el terrorismo moderno ha tenido pocos logros en objetivos estratégicos, pues los únicos casos claros son la expulsión del gobierno colonial de Gran Bretaña y Francia de Palestina, Chipre, Adén y Argelia (Wilkinson \& Mari, 2008).

En este segundo ciclo, los terroristas sabían bien que necesitaban de otros calificativos lingüísticos que no dieran lugar a connotaciones peyorativas como "terroristas", pues esta última les generaba imposibilidades legítimas de actividad política. En ese sentido, el grupo israelí Lehies fue el último en autodenominarse "terrorista". También la cabeza de Irgun ${ }^{3}$ comenzó a describir a los miembros de su organización como "libres combatientes" luchando contra los "gobiernos del terror" (Rapoport, 2004).

Otro punto clave y diferenciador entre las dos olas anteriores es que, en la segunda, las tácticas terroristas cambian sustancialmente. Las nuevas estrategias y tácticas eran más complejas que la simple planificación de asesinatos a líderes políticos de la primera ola porque existían más objetivos de alto valor por escoger. Sabían perfectamente que debían eliminar los sujetos del Estado como policías que fungían como ojos y oídos del imperio, así que también orquestaron secuestros sistemáticos a sus familiares (Rapoport, 2004). La estrategia era clara, "golpear y correr". Actuaban en pequeños grupos contra instalaciones policiales y militares, un primer acercamiento a la modalidad de las guerrillas en África, Asia y América Latina. Esta es la estrategia de la guerra de guerrillas.

Rapoport ha clasificado la tercera ola o "la nueva izquierda" como la que mejor refleja la dinámica de la Guerra de Vietnam. Una evidente asimetría en términos de capacidades militares convencionales y un desequilibrio de fuerzas estratégicas, son los focos de análisis del desenvolvimiento de la misma. Con ella se gestó una internacionalización de los métodos, usos y movimientos que empezarían a mezclarse con las causas guerrilleras. Se expandió por todo el mundo; empezó

3 Fue una organización sionista que operó durante el mandato británico de Palestina, entre los años de 1931 y de 1948. 
a estancarse y decayó en los 80 (DerGhounkassian, 2013). En ese orden de ideas, las guerrillas adoptaron elementos comunes terroristas aprendidos de Vietnam, en aras de lograr sus cometidos.

En esta ocasión el elemento de los nacionalismos también es crucial. Sin embargo, como tercera ola esta aumenta en su nivel, impacto y estrategias tácticas empleadas. La Guerra de Vietnam fue el conflicto -en términos geopolíticos y estratégicos- más importante de la posguerra. Modificó las relaciones entre militares y civiles, y entre políticos y periodistas. Puso en evidencia los límites del uso de la fuerza y transformó las relaciones entre bloques de la Guerra Fría (Sahagún, 2014).

Una de las principales características de este ciclo del terrorismo es que las actividades tuvieron lugar en la periferia de la Guerra Fría, la cual generó enormes ambivalencias en la estabilidad del sistema internacional. Las zonas de influencia de las dos potencias eran campos de batallas de diferentes intensidades. Los planes terroristas empezaron a concentrar estrategias de secuestros selectivos, toma de rehenes clave, como por ejemplo en 1979 el secuestro del ex primer ministro de Italia Aldo Moro, por parte de las Brigadas Rojas ${ }^{4}$; ante este hecho, el gobierno se rehusó a negociar y Moro fue brutalmente asesinado y su cuerpo esparcido por las calles (Rapoport, 2004).

El impacto psicológico empezaría a tomar un gran protagonismo en las actividades terroristas. Sería una de las piezas clave en el engranaje de la fundamentación del terror. En este espectro de la clasificación, la evolución de la amenaza, la mutación de su naturaleza e inclusive la popularización conceptual empezó a determinar con precisión lo que los Estados y regímenes entendían por terrorismo.

Cuando tuvo lugar el asesinato del embajador británico en Irlanda en 1976, el atentado contra Tatcher en 1984, contra el rey Hussein de Jordania, entre otros, el terrorismo se convirtió en un elemento contra

4 Grupo terrorista italiano de extrema izquierda fundado en 1969 que buscaba implantar un Estado socialista. Las Brigadas Rojas se especializaron en secuestros "express" y selectivos a altos funcionarios del gobierno y empresarios de Italia como objetivos de alto valor estratégico. Luego de una estrategia conjunta y combinada entre los servicios de seguridad franceses e italianos, el grupo tuvo una desactivación importante hacia 1989 trayendo consigo el proceso de disociación para la reincorporación de los miembros a la vida civil. 
el establishment. No obstante, los Estados asumían que era una amenaza efectiva cuando erosionaba la normalidad institucional.

Así las cosas, la envergadura de la sistematización de los actos, no solo en Europa, sino en América Latina y en otros espacios poco nombrados en las dinámicas mundiales, logra matricularse como un fenómeno internacional. En estas áreas se pusieron en marcha planes altamente codificados por los miembros de los grupos. Por ejemplo, para concluir la lógica de Vietnam, se orquestó la ofensiva del Tet. Un método estratégico que pretendía vislumbrar la fortaleza militar de Vietnam del Norte sobre el Sur y que cumplió su cometido de sembrar terror, en parte, gracias a su planeación en una importantísima campaña de distracción. A simple vista se puede considerar como el desenvolvimiento de una campaña de guerra asimétrica, pero dentro de la arquitectura asimétrica caben perfectamente metodologías como las del terrorismo.

Y por último, Rapoport expone una cuarta ola llamada la "ola religiosa”. Esta logra tener elementos más complejos que deben analizarse quirúrgicamente. El factor religión es, en muchas ocasiones, un motor de actividades humanas que pretende alcanzar algo divino. Esta dimensión soporta muchas acciones en nombre de un dios o un ente extrahumano. No obstante, este escenario aplica solo para algunas zonas y contextos, porque hay una amalgama entre lo religioso y lo étnico que en últimas logra una característica compleja.

Rapoport ha pensado estratégicamente en el mundo islámico cuando centra su análisis en la cuarta oleada. Su base analítica está enfrascada en descifrar epistemológicamente el devenir del terrorismo en Irán y su reflejo en Afganistán, así como los focos de su trabajo en Medio Oriente y las estepas de este fenómeno en África. De tal manera que Europa y Estados Unidos se han convertido en la cabeza de playa como objetivos y blancos de esta cuarta oleada del terrorismo.

El terrorismo religioso no es exclusivo de la teología islámica. Por ejemplo, los budistas en Sri Lanka intentaron transformar un escenario interno usando el terror de los tamiles con la intención de imponer un Estado secular (Rapoport, 2004). También se puede aludir a un terrorismo cristiano, si se tienen en cuenta las hazañas de ellos en la Edad Media con quienes no eran cristianos. Sin embargo, según el profesor Rapoport, el Islam es el corazón de esta ola. 
De hecho, el único que constituye una amenaza global proviene de la vertiente sunnita del fundamentalismo islámico. Hasta las organizaciones radicales musulmanas que operan en un contexto territorial definido se diferencian de Al Qaeda (DerGhounkassian, 2013) y no comparten su visión de futuro apocalíptico (Sivan, 2003). En ese orden de ideas, la religión es un factor determinante en la comprensión de este ciclo. Además se convierte, al mismo tiempo, en un objeto adicional dentro del estudio de las relaciones internacionales. Los atentados del 11 de septiembre han marcado un punto de inflexión en la historia contemporánea. Desde esos hechos se ha articulado, entre otras, una narrativa del nuevo barbarismo (Iranzo, 2006).

En últimas, este punto de inflexión marcó un desarrollo teórico, estratégico, político, jurídico y militar de lo que se entiende por terrorismo actualmente. Luego de decantar y reflexionar sobre las olas de Rapoport, es viable mencionar que el contexto actual representa una "marea alta" que integra las olas anteriores y suma nuevos elementos simultáneos y sistémicos del devenir del terrorismo.

Después de analizar las cuatro olas anteriores, y teniendo en cuenta la volatilidad de los conflictos, así como la preocupación de los Estados en cuanto a la seguridad internacional, la marea alta significa que la activación de las diferentes formas de terrorismo pueden ocurrir en tiempos contemporáneos, motivados por la religión o por los factores de los cuatro ciclos ya mencionados.

\section{La marea alta: la quinta ola, el ciclo incluyente}

Esta nueva categorización del ciclo del terrorismo, hace parte de un constructo adicional para lograr descifrar nuevas interpretaciones sobre el fenómeno. Este intento por mencionar una nueva etapa abandona la línea anterior del profesor Rapoport e intenta dimensionar nuevos elementos para el análisis. La quinta ola suma las cuestiones de las anteriores etapas, sin importar la temporalidad, y adicionalmente se 
esfuerza por explicar la inclusión de factores estratégicos y un nuevo elemento que termina alimentándola: el crimen organizado.

En varias ocasiones, la opinión pública confunde el crimen organizado con el terrorismo. Estos son dos fenómenos distintos aunque, cuando de factores ilegales se trata, comparten elementos al interior de sus actividades. Esta quinta ola, o como se ha denominado en este documento, la marea alta, es la mixtura y relación especial entre el crimen organizado y el terrorismo.

Si bien ambas metodologías ilegales (crimen organizado y terrorismo) contemplan grados y lógicas de violencia similares, persiguen diferentes fines. Salvo contadas excepciones, todo crimen involucra cierto grado de organización, de hecho, es organizado por naturaleza (Resa, 2014). El crimen organizado tiene un fin netamente lucrativo, pero así como el terrorismo tiene el vacío conceptual, el crimen organizado también tiene el privilegio de no tener una sola definición consensuada sobre su naturaleza y alcance. A pesar de lo anterior, la Convención de Palermo ha planteado la siguiente definición:

Grupo estructurado de tres o más personas que exista durante cierto tiempo y que actúe concertadamente con el propósito de cometer uno o más delitos graves o delitos tipificados con arreglo en la presente Convención con miras a obtener, directa o indirectamente, un beneficio económico u otro de orden material (Organización de las Naciones Unidas, 2000, p. 19).

El crimen organizado es un concepto de origen periodístico, que nunca alcanzó una satisfactoria definición criminológica, pero que se trasladó a la legislación penal y procesal para aumentar el ejercicio del poder punitivo respecto de un conjunto de delitos no bien delimitados (Zaffaroni, 2014). El crimen, per se, es organizado. Así sea de manera rudimentaria y sencilla.

Es importante subrayar que el crimen organizado, debido a su evolución y perfeccionamiento, tiene una importante cohesión interna en los Estados con altos grados de especialización y sofisticación. Cabe resaltar que el crimen organizado no es ideológico y tampoco busca el poder político como tal (Egenhoff \& Stein, 2011); sin embargo, logra 
acaparar algunas instituciones, funciona contaminándolas; su intención, en principio, no es poner en jaque al Estado, aunque su naturaleza clandestina los tipifica delinquiendo paralelamente al Estado. El crimen organizado es una actividad empresarial ilegal (Moreno, 2014).

La diferencia con el terrorismo es que este es el uso sistemático de la intimidación coercitiva, por lo general al servicio de intereses políticos. Es un método (Wilkinson \& Mari, 2008) que no tiene por naturaleza fines lucrativos, pero que busca, entre otras, poner en jaque a las instituciones.

Este tipo de amenazas que socaban el statu quo, comparten el privilegio de no ser claramente identificadas a nivel internacional. Si bien hay convenciones, declaraciones y resoluciones sobre cada una de ellas, no alcanzan a controlar de manera universal dichos fenómenos. El crimen y el terrorismo tienen un engranaje sistémico que los hace, en algunas ocasiones, inmunes a las estrategias de los regímenes internacionales. Su naturaleza clandestina y sinérgica hace que evolucionen y avancen a más velocidad que las estrategias de control y prevención de los Estados y de los esfuerzos multilaterales.

El terrorismo sabe bien que necesita de financiación, y el crimen es el método más eficiente para lograrlo. Gracias a esa armonía oscura y a los elementos de la marea alta es que cada vez es mucho más difícil, para los operadores de seguridad de los Estados y las instituciones internacionales, determinar si efectivamente lo sucedido es producto de un acto terrorista o tiene que ver con el crimen en su conjunto. En últimas, más allá del vacío conceptual existe un vacío estratégico sobre cómo actuar y enfrentarlos. Pero sí es evidente que la sumatoria de factores comunes y compartidos entre el crimen y el terrorismo ha hecho que se gesten particularidades de grandes andamiajes institucionales. Es decir, la red de funcionamiento se consolida cada vez más y mejor, hecho que genera un robustecimiento de sus actividades criminales y terroríficas. Sus alianzas estratégicas saben que la formación de canales, vías, nodos y paradigmas de funcionamiento evitan la intervención directa de los regímenes legales. Ponen en aprietos cualquier iniciativa.

La quinta ola es el ciclo incluyente. Este periodo no excluye modalidades, actores ni coyunturas. La religión, los ánimos separatistas, 
el antiimperialismo, el narcotráfico, el lavado de activos, las redes oscuras, los asesinatos masivos y selectivos hacen parte del gran saco de la quinta ola. El amalgamiento entre el crimen y el terrorismo hace que ambos sobrevivan de manera exitosa.

La coexistencia del terrorismo con el crimen organizado es viable gracias a los espacios vacíos de los Estados, las cañerías geográficas y al dinamismo subterráneo que convocan las prácticas delictivas. Esa compatibilidad se genera gracias a los espacios ingobernados (Naim, 2003) naturalmente y a los ingobernados artificialmente. Los primeros se refieren a los escenarios no controlados por fuerzas y operadores regulares u oficiales, como consecuencia de la geografía y los costos administrativos del Estado; mientras que los segundos se refieren a los espacios no controlados por las autoridades del Estado, a causa de la dinámica social, política, económica y de conflicto. Esos vacíos espaciales son nicho perfecto de retaguardia estratégica tanto del crimen organizado como del terrorismo. A continuación se presentan algunos ejemplos.

Tabla 1. Espacios ingobernados

\begin{tabular}{|l|c|c|}
\hline \multicolumn{1}{|c|}{ Espacio } & Natural & Artificial \\
\hline Bangladesh & & $\mathrm{x}$ \\
\hline Haití & $\mathrm{x}$ & $\mathrm{x}$ \\
\hline Somalia & & $\mathrm{x}$ \\
\hline República Centroafricana & & $\mathrm{x}$ \\
\hline Gaza & & \\
\hline Región del Darién & $\mathrm{x}$ & $\mathrm{x}$ \\
\hline Transnistria & & \\
\hline
\end{tabular}

Fuente: elaboración propia.

Los espacios anteriormente clasificados, responden a la ejemplificación categórica sobre regiones ingobernadas. No obstante, permanece abierto el debate sobre la condición situacional de dichas zonas, sus causas y naturalezas estructurales. De manera contundente se puede aseverar que en estos espacios coexisten el crimen organizado y el terrorismo. Sin duda alguna, los operadores de seguridad de los Estados 
se ven enfrentados a grandes retos y desafíos en la dinámica del control, vigilancia y prevención.

Estos espacios ingobernados son manejados como santuarios para el acopio, transporte y disposición de drogas ilícitas, trata de personas y vertimiento de residuos tóxicos, entre otros (Pérez, 2007). A diferencia de la exclusividad en la modalidad de ataque del terrorismo, el crimen organizado emplea la violencia como último recurso para alcanzar sus fines, lo que la convierte en una forma de vida (Bailey \& Godson, 2000). El terrorismo necesita del crimen para sostenerse y mantener una institucionalidad subterránea. Adicionalmente, los espacios ingobernados no solo están determinados exclusivamente en al análisis y bajo la actividad estatal. También existen espacios generados por la separación jurídica y política del crimen y el terrorismo.

En ese orden de ideas, la intensa cobertura mediática dedicada a la guerra contra el terrorismo oculta fenómenos globales similares, que los gobiernos pasan por alto a pesar de que son amenazas muy bien financiadas (Naim, 2003). En últimas, esos espacios pueden darse en diferentes dimensiones.

\section{Un espacio semántico vacío: indeterminación del término y efectos jurídicos}

Cuando de terrorismo se trata, los análisis de académicos y políticos están a la orden del día, así como los intentos por conceptualizar el fenómeno por parte de las instituciones, los regímenes internacionales y los Estados. Más allá de llegar a un consenso preliminar sobre la dimensión de la violencia (Soriano, 2007) como elemento fundamental del terrorismo, los instrumentos son insuficientes.

A pesar de la insuficiencia del estudio sistemático de este fenómeno de violencia en circunstancias de vieja data, los años 70 son un punto de inflexión en el cual se presenta un origen más reciente y robusto de carácter epistemológico sobre el terrorismo. El interés académico como fuente de debate, planeación y dimensión estratégica se da en la 
década de los años 70 debido al enorme aumento de las acciones terroristas de la década anterior. En ese orden de ideas, los objetivos de la moderna investigación van cambiando y mutando, pero el fenómeno terrorista sigue siendo un elemento problemático de investigación, sobre todo si nos acercamos a uno de los aspectos más controvertidos del mismo como es su definición.

Hasta ahora ni la jurisprudencia ni la doctrina se han puesto de acuerdo acerca del término terrorismo, tampoco lo ha hecho la ONU (Torres H. , 2010) o los Estados, aunque cada uno interpreta a su manera la amenaza y el terrorismo es lo que los actores hacen de él. Esa falta de acuerdo es lo que mantiene bloqueado el proyecto de Convenio sobre la materia en el seno de las Naciones Unidas (Conde \& Sara, 2012). Por ejemplo, en Estados Unidos existen diferentes definiciones elaboradas por el Departamento de Estado, el Departamento de Defensa, el FBI, la CIA y finalmente el Departamento del Tesoro.

Eso es reflejo de un gran vacío de interpretación, o tal vez de estrategia por entender qué es el terrorismo. El terrorismo tiene una inseguridad que lo hace seguro. La falta de definición lo blinda semántica y jurídicamente ante las dinámicas internas de los Estados y las fuerzas motrices del sistema internacional.

Esa indeterminación semántica es un escenario perfecto para elaborar en la subalternidad nodos estructurales que permitan la supervivencia del fenómeno. Pero la complejidad va mucho más allá y el terrorismo, el acto terrorista, y el terrorista son conceptos confusos que ocasionan que la coerción hacia quien vaya dirigida la acción de la justicia, sea más o menos mitigada. La valoración que haga quien tenga en su deber aplicar justicia al caso en concreto tiene varios problemas en la interpretación sobre lo que es y no es (Torres, 2010).

El vacío categórico, explicativo y conceptual no es una preocupación nueva. Por ejemplo, como se analizó al principio del documento con las olas de Rapoport, el fenómeno es cíclico y con grandes espacios libres de interpretación.

En esa misma línea, entre finales del siglo XIX y principios del XX nadie parecía estar a salvo de los ataques terroristas (Laqueur, 2002). Según Walter Laqueur (2002), el terrorismo se convirtió en la principal preocupación de políticos, jefes de policía, periodistas y escritores 
(desde Dostoievski a Henry James), a partir del asesinato del presidente francés Sadi Carnot, en 1894; el del primer ministro español Antonio Cánovas del Castillo, en 1897; la muerte a puñaladas de la emperatriz de Austria Isabel de Wittelsbach, por parte de un grupo de anarquistas en 1898; el asesinato del rey Humberto I de Italia en 1900, y la muerte en 1901 del presidente de los Estados Unidos, William Mckinley.

Lo anterior dejaba anonadados a los gobiernos, periodistas, cronistas, diplomáticos y tomadores de decisiones. La categorización sobre la actividad terrorista era y ha sido una arena movediza. Las actuales definiciones del terrorismo no son suficientes para describir la magnitud del problema en el mundo (Laqueur, 2002), a pesar de su milenaria naturaleza.

No obstante, el profesor Laqueur se ha atrevido a lanzar una definición arriesgada que no deja de ser ambigua per se,

El terrorismo se ha definido como el empleo sistemático de la violencia o la amenaza de usarla por parte de entidades menores que un Estado, con la finalidad de sembrar el terror en la sociedad para debilitar o incluso derrocar a quienes detentan el gobierno y así producir un cambio político. A veces puede transformarse gradualmente en una guerrilla (aunque a diferencia de las guerrillas, el terrorismo no puede o no quiere tomar territorios ni mantenerlos bajo su poder) En su larga historia, el terrorismo tuvo múltiples rostros; hoy la sociedad no enfrenta un terrorismo sino muchos (Laqueur, 2002, p. 261).

Si bien la anterior definición tiene varios elementos para una conceptualización más robusta, aún es imprecisa. No obstante tiene un elemento clave sujeto de análisis: la dimensión de "muchos terrorismos". Esa dimensión es quizá la que permite que exista indeterminación en la categorización y definición del fenómeno.

El mundo tiene un solo terrorismo, no varios. Lo que varía es la modalidad, los actores, la puntualización en el tiempo y en el espacio, así como las estrategias. La indeterminación de término del terrorismo posee un gran obstáculo para el derecho y los regímenes internacionales. Se convierte en un espacio vacío difícil de llenar generando 
incertidumbre jurídica sobre los alcances e implicaciones del fenómeno. Empero, dentro de ese fenómeno hay sub-categorías que pueden de manera taxativa arrojar elementos de estudio.

Teniendo en cuenta las dimensiones anteriores, los efectos jurídicos en la indeterminación del concepto son varios y múltiples. En primer lugar, no tener un consenso semántico trae como consecuencia una serie de vicios prácticos a la hora de positivizar una normatividad internacional en la materia. En segundo lugar, y como consecuencia del primer aspecto, debido a que no se ha logrado materializar una doctrina concreta, se deja a la libre interpretación de los actores - en muchas ocasiones los Estados-entender lo que les parece que es terrorismo. Eso, sin duda, es una subjetividad jurídica que no permite una categorización universal. Al no haber una doctrina unificada sobre el tema, cada actor entiende a su manera la amenaza. En tercer lugar, otro efecto jurídico tras la indeterminación es que la heterogeneidad conceptual permite que cada actor moldee sus códigos penales para tipificar el fenómeno; aunque ese evento deja que - mientras se forman los códigos jurídicos que le harán frente- la amenaza evolucione hasta el punto de ser revaluada y revisada antes de materializarse la iniciativa jurídica.

\section{El terrorismo y sus divisiones: elementos de análisis}

A lo largo del capítulo se ha intentado hacer una crítica al sistema internacional en conjunto con un fluido diálogo entre diversos autores en la categorización del fenómeno del terrorismo. En síntesis, en este apartado se quiere reflexionar y demostrar que si bien existen diversas caras del terrorismo, el fenómeno es uno solo. Para ello es necesario abordar los elementos precisos de manera taxativa; y aunque estos elementos, a simple vista, parecen distintos unos de otros, al final solo constituyen facetas que, gracias al vacío jurídico, dejan al descubierto que la lucha contra el terrorismo parece ser en vano.

Uno de los principales desafíos al entrar a estudiar la fenomenología del terrorismo es otorgarle categorías de análisis. En ese orden, 
desde Occidente, efectivamente se ha intentado conceptualizar, categorizar, definir y enmarcar todo fenómeno que rodea los problemas y cuestionamientos. A pesar de que el intento puede parecer una manía académica, siempre será necesario armar esquemas y mapas definitorios, por lo menos de referencia para tener puntos de partida en los juicios e interpretaciones.

\section{Terrorismo de Estado}

Es necesario retomar el mito del minotauro enunciado al principio del texto. Pero esta vez bajo otra interpretación, con el mismo Cortázar en la leyenda griega, pues la versión del terror del minotauro en su obra Los Reyes ${ }^{5}$ es diferente. Para Cortázar la bestia del minotauro simboliza el hombre libre y Teseo el terrorista al servicio del Estado (Olloqui, 2003). En este cambio de visión de la clásica historia helénica, en palabras de Cortázar "Teseo va a matar al minotauro como un gánster del rey", se convierte entonces en uno de los primeros terroristas de Estado y no necesariamente en un héroe (Olloqui, 2003).

Para esta reflexión, es imperante resaltar también el año 1793 y sus alrededores cronológicos. Pues a partir de ese contexto, los futuros acontecimientos tanto teóricos como políticos, estratégicos e incluso conceptuales, tendrán una explicación histórica con repercusiones en el presente y el futuro.

Cuando a principios de junio de 1793 los representantes de la Gironda eran detenidos y arrestados y el poder pasaba al grupo antagónico de la Convención -la Montaña-, organizado y dirigido por el Club jacobino, un nuevo mito histórico acababa de nacer: el Terror, el extremismo político, el exceso revolucionario, la confianza ciega en la razón humana y en su capacidad para crear una sociedad a partir de una teoría política dada (Rodríguez, 1992).

5 Es el primer libro de Julio Cortázar publicado con su verdadero nombre. 
Más allá de las interpretaciones literarias e históricas, que sin duda arrojan unas primeras pinceladas sobre la fenomenología de los actos, es imperante tener presente otras dimensiones. El terrorismo de Estado es una más de las aristas del problema, y de igual manera existe también un déficit consensuado sobre su definición y naturaleza. Esta arista se encuentra dentro de los Estados, pero gracias a esa característica es que frente al derecho internacional sigue habiendo enormes dificultades en la categorización y penalización. Como consecuencia de esto último, los vacíos jurídicos son imperantes en la conexión del fenómeno entre sí cuando trasciende a lo internacional.

El terrorismo de Estado está descrito por algunos como:

Una forma de ejercicio del poder estatal cuya regla de conocimiento permite y/o impone, con miras a crear el temor generalizado, la aplicación clandestina, impredecible y difusa, también a personas manifiestamente inocentes, de medidas coactivas prohibidas por el ordenamiento jurídico proclamado, obstaculiza o anula la actividad judicial y convierte al gobierno en agente activo de la lucha por el poder (Garzón, 2001, p. 129).

En sí, la característica principal es que es el gobierno y sus actores son quienes manifiestan la voluntad de generar el terror sobrepasando la lógica del contrato social. El Estado Social de Derecho es quebrantado y violado en múltiples dimensiones, hasta el punto en el cual el ciudadano común es considerado un foco de peligro para la seguridad del Estado.

La complejidad del asunto del terrorismo de Estado es de tal tenor que es necesario advertir que no existe un tipo penal que se denomine "terrorismo de Estado" (Torres, 2010). Quiere decir que el vacío jurídico dentro y fuera de los Estados es el hábitat plausible para la existencia del fenómeno. Eso implica una deficiencia estructural pero también una oportunidad para el terrorismo de gestarse y evolucionar sin spoilers ${ }^{6}$ inmediatos.

$6 \mathrm{Al}$ interior de las relaciones internacionales, el término spoiler se refiere a elementos, actores o circunstancias que actúan como "reventadores" que intentan bloquear una iniciativa o fenómeno. 
Si bien existe una indeterminación del término y concepto de terrorismo, con mayor lógica lo es también para el de Estado. En esa medida y bajo la lógica del terrorismo de Estado, es el gobierno quien, de manera no estructural, encasilla al enemigo y lo bautiza con el rótulo de terrorista. En últimas, la ecuación se invierte y no es el fenómeno per se quien determina la violencia contra el Estado, es el Estado quien adopta la metodología contra los ciudadanos ${ }^{7}$.

Siguiendo la anterior premisa, es necesario anotar que el empleo del terror por parte del Estado para combatir a una organización terrorista o a una amenaza de tal envergadura, es básicamente una confesión de la debilidad estratégica y estructural de dicho Estado como ente garante de la seguridad y la estabilidad. Es evidencia de una fragilidad del contrato social interno porque no es capaz de persuadir por medios legales y convencionales, llevando sus actividades a la represión, a la actividad delictiva que rompe cualquier grado de confianza de la ciudadanía hacia la institucionalidad. Dicha actividad no convencional del Estado, que atenta contra toda naturaleza jurídica de la arquitectura del mismo, se le ha denominado de distintas maneras: guerra sucia, terrorismo interno, guerra subterránea, acciones extrajudiciales, guerra irregular. Todas ellas responden a una debilidad y ruptura tanto del orden como de las instituciones.

\section{Terrorismo internacional $v s$ transnacional}

En el apartado anterior se hacía énfasis en la metodología del terrorismo dentro del Estado. Pero cuando de terrorismo internacional y transnacional se trata, la dinámica es diferente.

Esta clasificación del fenómeno se presta para diversas y equivocadas interpretaciones. Si se ha visto en este documento que la indeterminación del término es un obstáculo para precisar las dinámicas

7 Tener en cuenta los casos de las dictaduras del Cono Sur, el régimen franquista e incluso los sistemas teocráticos de Medio Oriente. 
terroristas, esta categoría logra ser el espacio perfecto para que dicha indeterminación funja en la complejidad conceptual.

Así las cosas, como bien lo expresa el profesor Reinares, el terrorismo internacional no es lo mismo que el terrorismo transnacional. $\mathrm{Ni}$ tampoco terrorismo internacional equivale a terrorismo islamista (Reinares, 2005). Lo anterior como unidades quirúrgicas en el análisis que compete esta investigación. Esta categoría implica un esfuerzo más amplio y complejo que cualquier otro, precisamente porque desde esta parten varios elementos que incidirán estratégicamente para el análisis posterior sobre el terrorismo como un régimen internacional subterráneo.

A menudo se alude al terrorismo internacional en términos vagos e imprecisos (como la noción de terrorismo genérico), lo que dificulta tanto una correcta apreciación de su alcance y dimensiones como un estudio cuidadoso de las tendencias (Reinares, 2005). Algo que ha sido imperante a lo largo de los siglos y de los Estados como agentes del sistema en la ardua tarea de la determinación conceptual, incluso con alcance jurídico.

Es frecuente confundir terrorismo transnacional y terrorismo internacional cuando el primero incluye al segundo, aunque esto no significa que el segundo tenga inmerso al primero como si fuera una sumatoria de factores comunes (Reinares, 1998).

Terrorismo transnacional es el que de una u otra manera atraviesa fronteras estatales, y quienes lo ejecutan, mantienen estructuras organizativas o desarrollan actividades violentas en más de un país, incluyendo por lo común territorios sobre los cuales no tienen jurisdicción alguna las autoridades a quienes dirigen en última instancia sus demandas. Esto significa que los actos de violencia involucran a más de un país y con frecuencia a individuos de dos o más nacionalidades, tanto por lo que se refiere a los terroristas como a sus víctimas (Reinares, 1998, p.32).

Mientras que la codificación de lo que es el terrorismo internacional involucra el objetivo y la estructura así: 
A primera vista, los criterios en atención a los cuales cabe delimitar como tal dicho fenómeno serian básicamente dos, uno relacionado con los objetivos que se persiguen mediante la práctica del terrorismo y otro referido a la extensión efectiva de las estructuras organizativas o redes que desarrollan esa violencia. Terrorismo internacional es, en primer lugar, el que se practica con la deliberada intención de afectar a la estructura y distribución del poder en regiones enteras del planeta o incluso a escala misma de la sociedad mundial. En segundo término, aquel cuyos actores individuales y colectivos hayan extendido sus actividades por un significativo número de países o áreas geopolíticas, en consonancia con el alcance de los propósitos declarados (1998, p. 32).

En últimas, categorizaciones como las dos anteriores son elementos de análisis para lograr determinar, dentro de la indeterminación, la compleja estructura, los modos, los actores y agentes que hacen del fenómeno un instrumento del sistema internacional de manera subterránea.

\section{Estatización del terrorismo}

Luego de los marcos de referencia anteriormente descritos, es imperante tratar de definir el papel del Estado en esta lógica del terrorismo. Así como se puso de manifiesto que el Estado es víctima en varias ocasiones de la fenomenología del terrorismo, también es receptor de la intensidad de la violencia, incluso puede fungir como un socio, artífice y generador de terrorismo.

El terrorismo es también una interpretación del Estado. Lo anterior puede redundar en una caracterización bastante constructivista. En ese sentido, en este documento se ha advertido constantemente que los vacíos conceptuales y la indeterminación jurídica son características persistentes cuando se aborda el tema. En otras palabras, es el Estado quien busca hacer del terrorismo un enemigo tangible para combatirlo.

Los analistas internacionales, las instituciones multilaterales y quienes toman las decisiones en los Estados critican sistemáticamente la falta de determinación, el bajo consenso y la carencia de instrumentos 
jurídicos internacionales para combatir ese flagelo. Como consecuencia de esto los mismos gobiernos han tenido que construir sus propios modelos y definiciones, con base en sus interpretaciones de lo que entienden por terrorismo. En otras palabras, han tenido que estatizar el terrorismo.

Esa estatización depende del constructo que el Estado elabore con base en sus propias experiencias, sus propios actores y, en efecto, sus dilemas internos de seguridad. Esa manifestación de lo que él y sus actores entienden por terrorismo suele encontrarse en los códigos penales, en los cuales se logra identificar una definición aún más puntual que la genérica emitida internacionalmente, los castigos, cargos y procedimientos legales del Estado. Son, en últimas, interpretaciones y asimilaciones de los Estados para hacer de sus códigos doctrinas a la medida del derecho penal, incluso con alusión al derecho penal del enemigo ${ }^{8}$.

Las cuatro olas del terrorismo, más la quinta denominada en este texto como la marea alta, en sincronía con las perspectivas analíticas de los diversos autores, logran arrojar un marco preliminar sobre la intencionalidad de determinar la dinámica del funcionamiento del terrorismo. Si bien no es una tarea fácil por los vacíos conceptuales y jurídicos internos, externos, y en ocasiones intermésticos, es relevante definirlos en aras de la interpretación, la doctrina y las políticas. Como se anunció al inicio de este libro, el fenómeno no es nuevo, pero el concepto sí.

Robert Pape crea una serie de tipologías sobre el terrorismo como fenómeno. Para el autor estadounidense, el terrorismo tiene tres grandes vertientes, que se manifiestan mediante: a) un terrorismo demostrativo, b) terrorismo destructivo y c) terrorismo suicida (Pape, 2003). Claramente, las tres dimensiones representan cuestiones estratégicas. En últimas, el terrorismo es un método estratégico.

8 Günther Jakobs acuñó el término afirmando que, para el poder penal del Estado no todos los ciudadanos son personas, sino que están "las personas y los enemigos". Estos últimos, que pueden ser tanto terroristas como violadores; en realidad no son considerados por el derecho como delincuentes, sino poco menos que animales peligrosos. (Jakobs \& Cancio, 2006), (Dozo, 2006). 
El terrorismo demostrativo es simplemente la concentración de capacidades que demuestran un grado determinado de zozobra capaz de poner en riesgo al actor referente. El terrorismo destructivo es la manifestación real de la potencia y capacidad de destrucción de un adversario, a través del método terrorista; mientras que el terrorismo suicida es la acción individual o colectiva de los miembros del grupo o red que pueden actuar con fines acumulados, es decir, el suicida intenta demostrar y destruir al mismo tiempo. Ni la fuerza militar ni la concesión territorial o política reducirá el terrorismo, gracias a esta última dimensión (Merke, 2005).

\section{Repensando al terrorismo: un dogma jurídico}

El terrorismo es uno de los fenómenos más estudiados y abordados por académicos, estrategas militares, políticos, etc. No obstante, el gran desafío es mitigar la indeterminación del concepto para entenderlo, pues gracias a eso es que, en cierta parte, es tan complejo de combatir. Así pues, repensar al terrorismo es un primer paso para restar ventaja frente a una amenaza que ya lleva un adelanto importante comparado a lo logrado por la convencionalidad.

La indeterminación del terrorismo no responde a un escaso abanico de conceptos, al contrario, ese vacío conceptual, en el constante repensamiento del fenómeno, es en últimas el que forja una indeterminación como la que ya se ha mencionado anteriormente. Al repensar la amenaza, es inevitable configurar una aproximación refiriéndose a que el terrorismo, según Walter Laqueur, no es una ideología sino la utilización de la violencia por elementos radicales y fanáticos de todo el espectro político (Pradera, 2003). Pero sigue siendo tan amplio, tan gaseoso y etéreo, que para Laqueur es inconveniente lanzar un asunto universal que no logra deconstruir su esencia.

El fenómeno del terrorismo es un dogma jurídico que se ha aceptado casi que por costumbre como amenaza tanto nacional como 
internacional. Su configuración dogmática ${ }^{9}$, como principio incuestionable refiriéndose a una amenaza innegable, precisa la necesidad sistémico-jurídica de modular su naturaleza, generar doctrina universal ante lo internacional y unificar criterios domésticos e internacionales. Así las cosas, la necesidad de repensar el terrorismo desnudando un dogma, son fundamentales a la hora de analizar, entender y precisar la intencionalidad de este trabajo de investigación por demostrar un nuevo enfoque científico e investigativo en la interpretación y entendimiento del fenómeno del terrorismo como un régimen internacional subterráneo.

No obstante, el dogma jurídico, aunque no tenga un cimiento unificado y estructurado sobre la amenaza del terrorismo, sí ha avanzado en un aspecto fundamental para intentar contrarrestar el funcionamiento del régimen subterráneo. Ese avance se ha materializado en 19 puntos marcos que fungen como instrumentos jurídicos universales contra el terrorismo, junto con las resoluciones del Consejo de Seguridad de las Naciones Unidas, sus convenios y protocolos que en últimas forman la estrategia global contraterrorista. Esos 19 elementos serán objeto de mención y análisis en este libro.

De lo anterior, se puede descifrar que en materia jurídica existen instrumentos que buscan eliminar y mitigar la amenaza, pero no entenderla. Eso es un fenómeno que va en contravía de toda iniciativa jurídica, si se tiene en cuenta que primero hay que conocer el fenómeno para entenderlo, observarlo, tipificarlo y luego sí elaborar y diseñar una serie de planes estratégicos para eliminarlo. En materia internacional, todo ha sido llevado a cabo de manera contraria permitiendo que el terrorismo — como fenómeno— también actúe de forma sistémica ocupando los espacios ingobernados que ha dejado el afán doctrinal por elaborar un concepto sin antes entenderlo.

9 Para la Real Academia de la lengua Española - RAE - , en su última versión No. 24, el concepto de dogma se refiere a que es una proposición que se asienta por firme y cierta y como principio innegable de una ciencia. Incluso, alude a un fundamento o puntos capitales de todo sistema, ciencia, doctrina o religión. 
En ese sentido, repensar el terrorismo incluye un nuevo eje de análisis en la interpretación del fenómeno y la amenaza. Si bien, en apartados siguientes, el centro de gravedad del documento girará en torno a la subterraneidad del terrorismo, es imperante determinar desde ahora que la metodología con la que se ha pensado el terrorismo debe tener un componente adicional, una variable más. En otras palabras, deben incluirse los antiguos y nuevos enfoques epistemológicos, jurídicos, e inclusive políticos del terrorismo como una cuestión de régimen internacional subterráneo.

En el esfuerzo por configurar el objeto de estudio, repensarlo y otorgarle un nuevo enfoque, es necesario precisar que es en la Convención de Ginebra de 1937 donde se alcanza a encontrar una definición de actos de terrorismo, definición difícil de encontrar en algún instrumento jurídico (Aparicio, 2012); no obstante, la dimensión legal solo se limitaba a definir la noción de los actos, pero no del fenómeno como tal. Un primer error en una cadena de ellos en la identificación integral de la amenaza.

Romper con el dogma tradicional parece ser absurdo para los simples ojos convencionales y tradicionales. No obstante, cambiar el paradigma tanto jurídico como fenomenológico, implica alimentar el conocimiento con instrumentos plausibles, viables, novedosos y estratégicos para entender y dar respuestas concretas a unas simples preguntas, ¿cómo y por qué?

Así las cosas, las ideas fundacionales que inspiran el nacimiento del terrorismo moderno no son originales, es decir, no corresponden a una simple coyuntura actual, mientras que sí lo son su justificación ideológica y metodológica, que han ido evolucionando a lo largo de la historia moderna (Aparicio, 2012). También ha sido algo transversal la preocupación por los hechos y por buscar iniciativas que, en ocasiones, han quedado como simples buenas intenciones. El terrorismo, más allá de ser una amenaza a la seguridad internacional, también lo es para la doctrina del derecho internacional. 


\section{Conclusiones}

La amenaza del terrorismo no es tan nueva como parece. A lo largo del capítulo se ha expuesto que el terrorismo no corresponde concretamente a la tipología de nueva amenaza como algunos sectores - entre ellos, el académico, estatal e incluso operadores de seguridad- han advertido. El fenómeno del terrorismo ha logrado configurar diferentes perspectivas de análisis y, gracias a esa lógica, la apuesta por el presente trabajo de analizarlo desde otro punto de vista.

En este capítulo se comprobó, de manera fenomenológica, que el terrorismo es tan antiguo que pueden mencionarse sus inicios incluso desde dinámicas mitológicas. El concepto como tal, sin duda es un constructo moderno y lleno de vacíos de todo tipo, pero la amenaza data de mucho tiempo atrás. En ese orden de ideas, la apuesta de este primer apartado ha sido vislumbrar — de manera analítica- las características básicas de la amenaza y de la condición que ha venido surgiendo a raíz de las cuatro "olas". Los principales autores que abordan el tema han elaborado una serie de marcos conceptuales bastante pertinentes, que han dejado claro el devenir histórico a lo largo del tiempo.

Esta investigación ha querido proponer, entre otras, una ampliación a la categorización de las cuatro "olas” ya referidas. En consecuencia, se ha planteado una quinta. En ella se analizó la sinergia estratégica entre el crimen organizado y el terrorismo haciendo las salvedades conceptuales pertinentes y claves. De ese mismo modo, el planteamiento sobre los vacíos conceptuales es imperante en este análisis debido a los riesgos jurídicos en los que el sistema internacional y los regímenes internacionales corren constantemente. La indeterminación del terrorismo es un obstáculo para combatirlo y la amenaza se acomoda perfectamente en esos espacios "ingobernados jurídica y terminológicamente" socavando no solo la doctrina sino la seguridad internacional.

Para analizar lo expuesto líneas atrás, fue necesario hacer un balance sobre las diferentes divisiones académicas que se tienen sobre el terrorismo. La dinámica encaminada a la determinación y concepción de los Estados frente al objeto de estudio, es un factor fundamental a la hora de lanzar apreciaciones jurídicas para extrapolar las nociones 
integrales al sistema internacional. Así las cosas, el balance hasta el momento ha permitido lanzar algunas consideraciones especiales referentes al terrorismo. Desde el punto de vista de la presente investigación, se vio la necesidad de abordar de manera crítica la situación, es decir, se plantea un escenario para repensar el terrorismo y desnudar un dogma jurídico que ha bloqueado tanto el análisis como la estrategia contraterrorista. Bajo ese marco, se pudo determinar que desde 1937, aproximadamente, se llevó a cabo una iniciativa internacional en el seno de la Convención de Ginebra para abordar de manera conceptual los actos del terrorismo. No obstante, la iniciativa ha quedado incompleta porque se preocupó por las consecuencias mas no por la configuración de la amenaza. No hay hasta el momento una definición que satisfaga de manera colectiva.

Este primer apartado es la punta de lanza para los enfoques y análisis posteriores de esta investigación. En ese sentido, si bien se ha hecho un esfuerzo por empezar a categorizar y darle cierta morfología al estudio, es necesario reconocer que las dificultades en la construcción de este capítulo radican en elementos fundamentales como el exceso de definiciones, las incorrectas maneras políticas de interpretar la fenomenología, la difícil extracción de la información de algunos sitios por ser de carácter restringido, hasta los distintos visos y matices en la materia desde las visiones occidentales. En ese orden, la multiplicidad de información, contra todos los pronósticos, también es una dificultad debido a la baja institucionalización del término y los bajos niveles de consenso en la materia.

El primer capítulo de este libro es el "abrebocas" de una apuesta investigativa novedosa: demostrar que el terrorismo ha configurado un régimen internacional subterráneo, una visión que hasta el momento no tiene un precedente académico. 
